\title{
Synchronization of simple chaotic flows
}

\author{
Lars Callenbach, Stefan J. Linz*, Peter Hänggi \\ Theoretische Physik I, Institut für Physik, Universität Augsburg, D-86135 Augsburg, Germany \\ Received 23 February 2001; received in revised form 22 June 2001; accepted 26 June 2001 \\ Communicated by C.R. Doering
}

\begin{abstract}
Nonlinear scalar third-order differential equation or jerky dynamics $\dddot{x}=J(x, \dot{x}, \ddot{x})$ have recently attracted considerable interest since they constitute an important tool to identify and classify elementary chaotic flows. We investigate whether and under what conditions such systems can be synchronized by various coupling schemes such as the methods of PecoraCarroll and Cuomo-Oppenheim, BK-coupling and active-passive decomposition. In particular, for the latter two schemes, we present specific, simplified coupling or decomposition approaches that allow for analytical estimates of the rapidity of the synchronization error. (C) 2001 Elsevier Science B.V. All rights reserved.
\end{abstract}

PACS: 05.45.Xt; 05.45.Ac; 82.40.Bj

Keywords: Chaos; Synchronization; Jerk; Flow; Differential equation

\section{Introduction}

In the broadest sense, synchronization terms the tendency of two or more appropriately coupled dynamical systems to undergo resembling evolution in time. As shown in a seminal paper by Pecora and Carroll [1] in 1990, synchronization can also be achieved if the uncoupled dynamical systems exhibit chaotic timeevolution. Their basic idea was to take two (identical) replicas of a three-dimensional dynamical system $\dot{\mathbf{x}}=$ $\mathbf{V}(\mathbf{x})$ with $\mathbf{x}=(x, y, z)^{\mathrm{T}}$ and $\mathbf{V}(\mathbf{x})$ being the vector field, to use one of them, the drive system $\dot{\mathbf{x}}_{d}=\mathbf{V}\left(\mathbf{x}_{d}\right)$, as an unconstraintly developing system and to couple the second one, the response system $\dot{\mathbf{x}}_{r}=\mathbf{V}\left(\mathbf{x}_{r}\right)$, unidirectionally to the drive system by a suitable replacement of the dynamical variables in the response system.

\footnotetext{
* Corresponding author.

E-mail address: linz@ physik.uni-augsburg.de (S.J. Linz).
}

The signature of synchronization is given by the time evolution of the difference between drive and response variables, commonly called the error $[1,2]$,

$\mathbf{e}=\mathbf{x}_{d}-\mathbf{x}_{r}$.

If $\mathbf{e}(t) \rightarrow 0$ as $t \rightarrow \infty$, the dynamics of the response system approaches the time evolution of the drive system and synchronization of these two systems is guaranteed. Pecora and Carroll [1] demonstrated such a behavior, e.g., by using the Lorenz flow and, as coupling scheme, (i) identical dynamics of $x_{d}$ and $x_{r}$, and (ii) replacement of $x_{r}$ by $x_{d}$ in the $y$ and $z$ components of the vector field of the response system.

Achieving synchronization between two chaotic systems, however, is far from being straightforward. It sensitively depends on the considered dynamical systems and the specific coupling method. In the wake of the study by Pecora and Carroll, a variety of alternative coupling schemes have been studied (for a review see [2]), among them the Cuomo-Oppenheim [3], BK- 
coupling $[2,4]$ schemes and the active passive decomposition (APD) [5-7], and also extensions to generalized synchronization of nonidentical chaotic systems [2]. Besides the Lorenz model, specific applications of synchronization have been mostly investigated for the Rössler model, the piece-wise Rössler model and Chua's oscillator [2].

On the other hand, there has been recently progress in identifying and classifying the elementary chaotic flows and finding necessary, albeit not sufficient criteria for the emergence of elementary chaotic dynamics. Here, the key point was to start from third-order differential equations $\dddot{x}=J(x, \dot{x}, \ddot{x})$ in one scalar variable $x(t)$ [8-15], commonly called jerky dynamics because of the direct mechanical interpretation of $\dddot{x}$ as time rate of change of the acceleration, $\dddot{x}=$ $J(x, \dot{x}, \ddot{x})$. Setting $y=\dot{x}$ and $z=\ddot{x}$, a jerky dynamics is equivalent to the vector field $\dot{\mathbf{x}}=\mathbf{V}(\mathbf{x})$ with $\mathbf{V}(\mathbf{x})=$ $(y, z, J(x, y, z))^{\mathrm{T}}$. Within the last several years, intensive numerical search $[9,12,15]$ and also analytical reduction techniques to jerky dynamics for known vector fields $[10,11]$ have lead to a fairly complete list of elementary functional forms of jerky dynamics that exhibit chaotic behavior in some parameter ranges. These systems are not only restricted to polynomial nonlinearities, but also cover piece-wise linear forms of jerk functions $J(x, \dot{x}, \ddot{x})$ that fulfill a Lipschitz condition, i.e., the corresponding vector field satisfies $\left|\mathbf{V}\left(\mathbf{x}_{1}\right)-\mathbf{V}\left(\mathbf{x}_{2}\right)\right| \leqslant L\left|\mathbf{x}_{1}-\mathbf{x}_{2}\right|$ with $L$ being a finite, positive constant. This guarantees local existence and uniqueness of the solutions of the considered systems. Particularly the piece-wise linear jerky dynamics $[12,13]$ are attractive candidates for synchronization since they are both functionally simple and easily realizable as electronic circuits [13].

The most elementary chaotic jerky dynamics with modulus nonlinearity is determined by the model equation by Linz and Sprott [12]

$\dddot{x}=-A \ddot{x}-\dot{x}+|x|-1$

that is chaotic, e.g., for $A=0.61$. It constitutes a minimal dissipative chaotic system in the sense that no term entering in (2) can be omitted without loosing potentially chaotic behavior [14]. The dynamics of (2) can also be electronically realized with high accuracy [13].

The purpose of this Letter is at least three-fold. First, taking a special form of a jerky dynamics $\dddot{x}=$
$J(x, \dot{x}, \ddot{x})$, we investigate whether the most popular synchronization methods, the Pecora-Carroll [1, 2] and the Cuomo-Oppenheim schemes [3] are applicable to chaotic jerky dynamics to obtain synchronization (cf. Section 2). Moreover, we show that the BK- and APD-coupling schemes do generally work (cf. Sections 3 and 4) for arbitrary jerky dynamics. Second, we derive general estimates for the decay behavior of simplified versions of the latter two schemes in order to determine criteria for effective, i.e., rather rapid synchronization. Third, we demonstrate our general results by applying it to and numerically validating it for the specific case of the minimal chaotic model with modulus nonlinearity, Eq. (2).

\section{Simple synchronization methods}

In this section we inspect whether the two most elementary synchronization schemes by Pecora and Carroll [1,2] and by Cuomo and Oppenheim [3] do work for jerky dynamics. Since a general discussion for arbitrary functional forms of jerky dynamics, $\dddot{x}=$ $J(x, \dot{x}, \ddot{x})$ does not seem to be feasible, we only consider here the important subclass of a jerky dynamics that only possesses nonlinear terms in $x$, i.e.,

$\dddot{x}=-A \ddot{x}-B \dot{x}+g(x)$

with $g(x)$ being a nonlinear function of $x$ only and Eq. (2) being a special case. For this class, an extensive list of functional forms of $g(x)$ and parameter values of $A$ and $B$ leading to chaotic behavior of Eq. (3) has been recently published [13] and also necessary (analytical) criteria for the appearance of dissipative chaos, most importantly $A>0$ and $B>0$, have been discovered [14].

Adapting the idea of the synchronization scheme of Pecora and Carroll [1], i.e., keeping the identical $x$-dynamics of the drive and the response system and replacing $x_{r}=x_{d}$ in the remaining $y_{r}$ and $z_{r}$ dynamics, one obtains for the drive system

$\dot{x}_{d}=y_{d}$,

$\dot{y}_{d}=z_{d}$,

$\dot{z}_{d}=-B y_{d}-A z_{d}+g\left(x_{d}\right)$,

whereas the response system takes on the form

$x_{r}=x_{d}$, 
$\dot{y}_{r}=z_{r}$,

$\dot{z}_{r}=-B y_{r}-A z_{r}+g\left(x_{d}\right)$.

Since the $x$-component of the drive and the response system undergo identical dynamics, only the subsystem of the $y$ and $z$ components have to be considered to obtain the error dynamics. This yields the autonomous linear system

$$
\begin{aligned}
& \dot{e}_{y}=e_{z}, \\
& \dot{e}_{z}=-B e_{y}-A e_{z} .
\end{aligned}
$$

Therefore, the error dynamics is determined by the eigenvalues $\lambda$ of the characteristic equation $[\lambda(A+$ $\lambda)+B]=0$ that are given by $\lambda_{y, z}=-A / 2 \pm$ $\sqrt{A^{2} / 4-B}$. Using the aforementioned necessary conditions for the appearance of chaos in system (3) $(A>0, B>0)$, we conclude that the real parts of both eigenvalues of the error dynamics are negative if Eq. (3) displays chaotic dynamics and, as a consequence, the class (3) can be synchronized by the Pecora-Carroll method.

Applying the Cuomo-Oppenheim scheme [3] to Eq. (3), i.e., keeping the drive system (4) and substituting $x_{r}$ by $x_{d}$ in the corresponding vector field of the response system, Eq. (5) is replaced by

$$
\begin{aligned}
& \dot{x}_{r}=y_{r}, \\
& \dot{y}_{r}=z_{r}, \\
& \dot{z}_{r}=-B y_{r}-A z_{r}+g\left(x_{d}\right) .
\end{aligned}
$$

The corresponding error dynamics is determined by the autonomous linear system

$\dot{e}_{x}=e_{y}$,

$\dot{e}_{y}=e_{z}$,

$\dot{e}_{z}=-B e_{y}-A e_{z}$.

As a consequence, the eigenvalues determining the growth/decay of the error $\mathbf{e}$ result from the characteristic equation $\lambda[\lambda(A+\lambda)+B]=0$. Since one eigenvalue is always equal to zero, chaotic synchronization of system (3) cannot be achieved by the CuomoOppenheim method. The modulus of the error $|\mathbf{e}|$ approaches a constant non-zero value as $t \rightarrow \infty$ that is determined by the initial conditions.

Although the Pecora-Carroll scheme is applicable to achieve synchronization of chaotic models of type (3), there are at least two drawbacks. First, there is no way to speed up the synchronization since the decay rate is bounded by the eigenvalue of (6) with the largest real part, and, therefore, directly determined by the control parameter combinations $A, B$ where (3) is chaotic. Second, focusing on applications to private communication, only one component of the drive system is transmitted. Even if an information $I(t)$ is superposed to $x_{d}(t)$, it might be possible to reconstruct the drive system because of the intimate relation of the drive variables $y_{d}=\dot{x}_{d}$ and $z_{d}=\ddot{x}_{d}$ with the transmitted signal $x_{d}$. To avoid these two drawbacks, we discuss in the next two sections two variants of synchronous coupling schemes that also apply to arbitrary jerky dynamics.

\section{BK-coupling}

The BK-coupling scheme [2] is a variant of a synchronous substitution scheme where several or all variables of the drive system are coupled to the response system. The general idea behind BK-coupling is as follows [2]: synchronization can be directly achieved if there is a negative feedback between drive and response system, i.e., $\dot{\mathbf{x}}_{r}=\mathbf{V}\left(\mathbf{x}_{r}\right)+c\left(\mathbf{x}_{d}-\mathbf{x}_{r}\right)$ with an appropriately chosen scalar constant $c$. Such a scheme, however, would require the transmission of all three components of the drive system $\mathbf{x}_{d}$. However, if the constant $c$ is replaced by a matrix of the specific form $\mathbf{B K}^{\mathrm{T}}$ with $\mathbf{B}=\left(B_{1}, B_{2}, B_{3}\right)^{\mathrm{T}}$ and $\mathbf{K}=\left(K_{1}, K_{2}, K_{3}\right)^{\mathrm{T}}$ being vectors with constant and so far arbitrary coefficients, the coupling term $\mathbf{B} \mathbf{K}^{\mathrm{T}}\left(\mathbf{x}_{d}-\mathbf{x}_{r}\right)$ can be rearranged to yield $\mathbf{B}\left(\mathbf{K} \cdot \mathbf{x}_{d}-\mathbf{K} \cdot \mathbf{x}_{r}\right)$. The latter shows that, in principle, only the scalar combination $\mathbf{K} \cdot \mathbf{x}_{d}$ needs to be transmitted to the response system to achieve synchronization. The major problem, however, is to find appropriate combinations of the six parameters in $\mathbf{B}$ and $\mathbf{K}$. The BK-coupling approach originally stems from control theory and it is known [16] that, in principle, it works for so-called Brunowsky canonical forms, i.e., $n$ th-order dynamical systems that can be directly rewritten as $n$ th-order differential equations in one scalar variable.

Specifically, for a jerky dynamics, the BK-coupling scheme is determined by the drive system

$\dot{x}_{d}=y_{d}$,
$\dot{y}_{d}=z_{d}$, 
$\dot{z}_{d}=J\left(x_{d}, y_{d}, z_{d}\right)$,

and the response system

$\dot{x}_{r}=y_{r}+B_{1}(s-\tilde{s})$,

$\dot{y}_{r}=z_{r}+B_{2}(s-\tilde{s})$,

$\dot{z}_{r}=J\left(x_{r}, y_{r}, z_{r}\right)+B_{3}(s-\tilde{s})$,

where $s=\mathbf{K} \cdot \mathbf{x}_{d}$ is the transmitted signal and $\tilde{s}=$ $\mathbf{K} \cdot \mathbf{x}_{r}$. The second term on the rhs of the response system is the linear feedback term that basically contains the weighted difference of $s$ determined by the drive system and $\tilde{s}$ by the response system.

Is there a simple and generally applicable choice of the coefficients of $\mathbf{B}$ and $\mathbf{K}$ that ensures synchronization for all types of jerky dynamics? In the remainder of this section, we will show by considering a representative example and using rigorous mathematical arguments that the specific form of the matrix $\mathbf{B K}^{\mathrm{T}}$,

$\mathbf{B K}^{\mathrm{T}}=\left(\begin{array}{ccc}a & 1 & 1 / a \\ a^{2} & a & 1 \\ a^{3} & a^{2} & a\end{array}\right)$,

or, equivalently, the choice $\mathbf{B}=\left(a^{0}, a^{1}, a^{2}\right)^{\mathrm{T}}$ and $\mathbf{K}=$ $\left(a^{1}, a^{0}, a^{-1}\right)^{\mathrm{T}}$, that contains only one adjustable, appropriately chosen real parameter a leads to a decaying error dynamics and, therefore, to synchronization.

As a representative example, we use in the coupling scheme (9) and (10) the jerk function $J(x, \dot{x}, \ddot{x})$ of the model equation (2) with the control parameter $A=0.61$ (where Eq. (2) displays chaotic behavior) and two distinct parameter values for $a$ entering into $\mathbf{B}$ and $\mathbf{K}$, namely $a=13$ and $a=42$. The numerical computation of the corresponding Euclidean length of the error dynamics $|\mathbf{e}|=\sqrt{e_{x}^{2}+e_{y}^{2}+e_{z}^{2}}$ as function of time is depicted in Fig. 1. In both cases we observe a rapid convergence of $|\mathbf{e}|$ to zero with time that decays oscillatory, but faster than exponential in time. For $a=$ 13 the decay rate is bounded by $\exp (-t)$, whereas for $a=42$ by $\exp (-10 t)$. Notably, the synchronization is faster, the larger the value of $a$ is. The fact that this type of synchronization works for these specific cases raises the questions whether it applies to any type of jerky dynamics and what restrictions on the value of $a$ hold.

To proof the general validity of the synchronization scheme for the afore-mentioned form of the matrix $\mathbf{B K}^{\mathrm{T}}$, Eq. (11), and any jerky dynamics, we consider

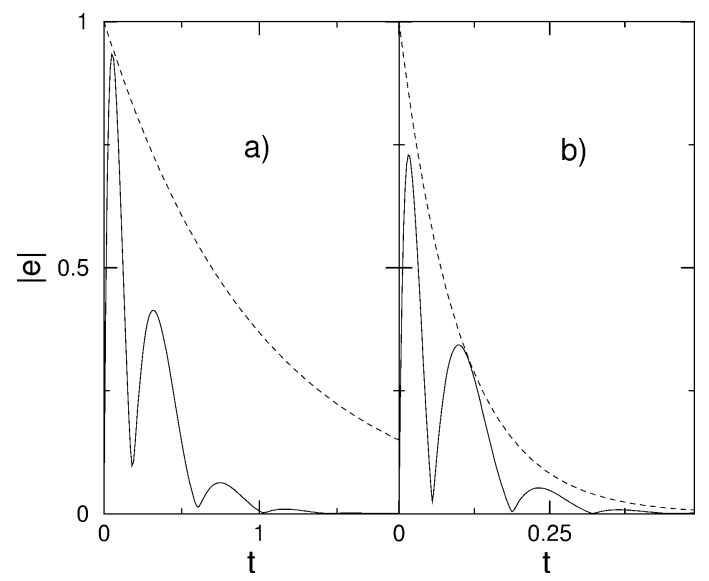

Fig. 1. Numerically computed time evolution of the synchronization error $|\mathbf{e}|$ for jerky dynamics coupled via the BK scheme, Eqs. (9) and (10), the chaotic jerky dynamics, Eq. (3) with $A=0.61$, and the two representative values of the $a$ in the BK-coupling scheme, (a) $a=13$ and (b) $a=42$. Dashed lines represent corresponding upper bounds of the error being proportional to $\exp (-t)$ in (a) and $\exp (-10 t)$ in (b).

its error dynamics of Eqs. (9) and (10) given by

$\dot{\mathbf{e}}=-\mathbf{B} \mathbf{K}^{\mathrm{T}} \mathbf{e}+\left(\begin{array}{c}e_{y} \\ e_{z} \\ J\left(\mathbf{x}_{d}\right)-J\left(\mathbf{x}_{r}\right)\end{array}\right)$.

Next, we use the trick to formally rewrite the third component of the second vector on the rhs of Eq. (12) as a scalar product of the error $\mathbf{e}$ and a vector $\left(J^{x}\right.$, $\left.J^{y}, J^{z}\right)^{\mathrm{T}}$ containing some real-valued functions that depend both on $\mathbf{x}_{d}$ and $\mathbf{x}_{r}$,

$$
\begin{aligned}
& J\left(\mathbf{x}_{d}\right)-J\left(\mathbf{x}_{r}\right) \\
& \quad=\left(J^{x}\left(\mathbf{x}_{d}, \mathbf{x}_{r}\right), J^{y}\left(\mathbf{x}_{d}, \mathbf{x}_{r}\right), J^{z}\left(\mathbf{x}_{d}, \mathbf{x}_{r}\right)\right)^{\mathrm{T}} \mathbf{e} .
\end{aligned}
$$

The functions $J^{x}, J^{y}, J^{z}$ are determined by

$$
\begin{aligned}
& J^{x}\left(\mathbf{x}_{d}, \mathbf{x}_{r}\right)=\frac{J\left(x_{r}, y_{r}, z_{r}\right)-J\left(x_{d}, y_{r}, z_{r}\right)}{x_{r}-x_{d}}, \\
& x_{d} \neq x_{r}, \\
& J^{y}\left(\mathbf{x}_{d}, \mathbf{x}_{r}\right)=\frac{J\left(x_{d}, y_{r}, z_{r}\right)-J\left(x_{d}, y_{d}, z_{r}\right)}{y_{r}-y_{d}}, \\
& y_{d} \neq y_{r}, \\
& J^{z}\left(\mathbf{x}_{d}, \mathbf{x}_{r}\right)=\frac{J\left(x_{d}, y_{d}, z_{r}\right)-J\left(x_{d}, y_{d}, z_{d}\right)}{z_{r}-z_{d}}, \\
& z_{d} \neq z_{r} .
\end{aligned}
$$

As a mathematical side-remark we note that if $J$ fulfills a Lipschitz condition on a subset $C \subset \mathbb{R}^{3}$ with 
a Lipschitz constant $L_{C}$, the functions $J^{x}, J^{y}$ and $J^{z}$ are continuous on $N=\left\{\vec{y}=\left(y_{1}, \ldots, y_{6}\right)^{\mathrm{T}} \in \mathbb{R}^{6}: y_{1} \neq\right.$ $\left.y_{4}, \quad y_{2} \neq y_{5}, \quad y_{3} \neq y_{6}\right\}$. Moreover, $J^{x}, J^{y}, J^{z}$ can be extended continuously 'from one side' approaching $\mathbb{R}^{6}-N$ and are bounded by $L_{C}$ on the bounded subset $C \times C \subset \mathbb{R}^{6}$. This implies that $J^{x}, J^{y}, J^{z}$ are bounded by $L_{C}$ in the limits $x_{d} \rightarrow x_{r}, y_{d} \rightarrow y_{r}$ and $z_{d} \rightarrow z_{r}$, respectively.

Using (14), we are in the position to rearrange Eq. (12) in the form

$\dot{\mathbf{e}}=\mathrm{Ae}+\mathrm{Be}$,

with

$$
\begin{aligned}
\mathbf{A} & =-\mathbf{B} \mathbf{K}^{\mathrm{T}}+\left(\begin{array}{lll}
0 & 1 & 0 \\
0 & 0 & 1 \\
0 & 0 & 0
\end{array}\right) \\
& =-\left(\begin{array}{ccc}
a & 0 & 1 / a \\
a^{2} & a & 0 \\
a^{3} & a^{2} & a
\end{array}\right)
\end{aligned}
$$

being a matrix with time-independent coefficients, and

$\mathrm{B}=\left(\begin{array}{ccc}0 & 0 & 0 \\ 0 & 0 & 0 \\ J^{x} & J^{y} & J^{z}\end{array}\right)$

containing time-dependent contributions via the dynamics of $\mathbf{x}_{d}$ and $\mathbf{x}_{r}$.

To proceed, we take advantage of the PoincareLyapunov theory for asymptotic stability [17] and modify it appropriately to find conditions to ensure synchronization. Calculating the eigenvalues of the matrix $\mathrm{A}$, given by $\lambda_{1,2} \approx-0.337 a \pm i 0.562 a, \lambda_{3}=$ $-2.324 a$ and the corresponding eigenvectors, one can construct the matrix $S$ that transforms $A$ into diagonal form $D=S^{-1} A S$. To estimate the time evolution of the error dynamics, we can take advantage of $S$ as follows. Multiplying (15) by $S^{-1}$ and introducing the transformed error dynamics $\tilde{\mathbf{e}}=\mathrm{S}^{-1} \mathbf{e}$ yields

$\dot{\tilde{\mathbf{e}}}=\mathrm{D} \tilde{\mathbf{e}}+\tilde{\mathrm{B}} \tilde{\mathbf{e}}$

with $\tilde{B}=S^{-1}$ BS. Formal integration of (18) leads to

$\tilde{\mathbf{e}}(t)=e^{\mathrm{D} t} \tilde{\mathbf{e}}(0)+\int_{0}^{t} e^{\mathrm{D}(t-s)} \tilde{\mathrm{B}}(s) \tilde{\mathbf{e}}(s) d s$

and, after taking the norm, to the inequality

$\|\tilde{\mathbf{e}}(t)\| \leqslant\left\|e^{\mathrm{D} t}\right\|_{M}\|\tilde{\mathbf{e}}(0)\|$

$$
+\int_{0}^{t}\left\|e^{\mathrm{D}(t-s)} \tilde{\mathrm{B}}(s)\right\|_{M}\|\tilde{\mathbf{e}}(s)\| d s,
$$

where $\left\|\mathrm{A}=\left(a_{i, j}\right)\right\|_{M}$ is the maximum of $\sum_{j=1}^{3}\left|a_{i, j}\right|$ of the rows of the matrix A. Since D is diagonal, the norm of $e^{\mathrm{D} t}$ is bounded by $e^{-q t}$, where $q$ is the eigenvalue of the matrix $A$ with the largest real part, i.e., $q<a / 3$. Therefore, (20) can be recast into

$$
\begin{aligned}
\|\tilde{\mathbf{e}}(t)\| \leqslant & e^{-q t}\|\tilde{\mathbf{e}}(0)\| \\
& +\int_{0}^{t} e^{-q(t-s)}\left\|\tilde{\mathrm{B}}^{\mathrm{T}}(s)\right\|_{M}\|\tilde{\mathbf{e}}(s)\| d s .
\end{aligned}
$$

Multiplying (21) by $e^{q t}$ and introducing the abbreviation $f(t)=e^{q t}\|\tilde{\mathbf{e}}(t)\|$, we obtain

$f(t)=\|\tilde{\mathbf{e}}(0)\|+\int_{0}^{t} f(s)\left\|\tilde{\mathrm{B}}^{\mathrm{T}}(s)\right\|_{M} d s$.

Since $f(t)$ is strictly positive, one can apply Gronwall's inequality [17] yielding

$f(t) \leqslant\|\tilde{\mathbf{e}}(0)\| \exp \left(\int_{0}^{t}\left\|\tilde{\mathrm{B}}^{\mathrm{T}}(s)\right\|_{M} d s\right)$.

Therefore, the transformed error dynamics fulfills

$$
\begin{aligned}
\|\tilde{\mathbf{e}}(t)\| & \leqslant f(t) e^{-q t} \\
& \leqslant\|\tilde{\mathbf{e}}(0)\| \exp \left(\int_{0}^{t}\left(\left\|\tilde{\mathrm{B}}^{\mathrm{T}}(s)\right\|_{M}-q\right) d s\right) .
\end{aligned}
$$

Using the supremum of $\left\|\tilde{\mathrm{B}}^{\mathrm{T}}\right\|_{M}, b=\sup _{t} \| \mathrm{S}^{-1} \mathrm{~B}(t) \times$ $\mathrm{S}\|\|_{M}$, the error dynamics is bounded by

$$
\|\tilde{\mathbf{e}}(t)\| \leqslant\|\tilde{\mathbf{e}}(0)\| \exp \left(\int_{0}^{t}(b-a / 3) d s\right)
$$

and vanishes asymptotically if $b-a / 3$ is negative. It remains to calculate $b$. An upper bound for $b$ can be found by splitting $B$ in a sum of three matrices which have each only one non-zero component (either $J^{x}, J^{y}$ or $J^{z}$ ), multiplying these by $\mathrm{S}^{-1}$ (from the left) and $\mathrm{S}$ (from the right) and using the fact that $J^{x}, J^{y}, J^{z}$ are bounded by the Lipschitz constant $L_{C}$. With the help of computer algebra one obtains that $b<4 L_{C}$ 
if the additional condition $a>1$ holds. Therefore, synchronization of (9) and (10) is guaranteed if

$a>\max \left\{12 L_{C}, 1\right\}$.

This is the central result of this section. Moreover, if one requests that the decay of the error should decay faster than $e^{-c t}$ with a prescribed constant $c>0$, then the stronger condition

$a>\max \left\{3\left(4 L_{C}+c\right), 1\right\}$

holds.

Several remarks are in order: (i) The different functions $J^{x}, J^{y}, J^{z}$ contribute in a different way to the constant $b$. Looking at one of them and neglecting the others we find the following behavior: $J^{x}: b \sim$ $L_{C} a^{-2}, J^{y}: b \sim L_{C} a^{-1}$ and $J^{z}: b \sim L_{C}$. For $a>1$, this reflects the fact that it is much more difficult to reduce a disturbance in $z$ than in $y$ or $x$. (ii) As a generalization, the above derivation of the choice of the parameter $a$ can be extended to other types of couplings that might be nonlinear. Although such a generalized coupling does not, in general, increase the speed of synchronization, it might be useful for potential applications of more secure communication schemes since it is more difficult to determine all relevant parameters using autosynchronization for parameter estimation [18]. For example, if one transmits a signal $s=p\left(\mathbf{x}_{d}\right)$ instead of $s=\mathbf{K} \mathbf{x}_{d}$ and uses the parameters $\left(B_{1}, B_{2}, B_{3}\right)=\left(1, a, a^{2}\right)$, the function $p$ only has to satisfy the conditions

$\left|p^{x} / a-1\right|,\left|p^{y}-1\right|,\left|a p^{z}-1\right| \leqslant L_{C} / a$

by using the linearized functions $p^{x}, p^{y}, p^{z}$ defined analogously to the $J^{x}, J^{y}, J^{z}$ in Eq. (14). If these conditions are satisfied we obtain the inequality $b \leqslant$ $(4+12) L_{C}$ with $b=\sup _{t}\left\|\mathrm{~S}^{-1} \mathrm{~B}(t) S\right\|_{M}$. Here, the term $4 L_{C}$ arises from the influence of $J$, whereas the term $12 L_{C}$ reflects the contribution of the nonlinear function $p$. With this bound for $b$ we then obtain the generalized estimate

$a \geqslant \max \left\{48 L_{C}+3 c, 1\right\}$

for the parameter $a$ if the error should decay faster than $e^{-c t}$. Looking at inequality (28) we can see that the influence of $p^{z}$, the disturbance of the 'second derivative', is larger than the disturbance coming from $p^{x}$ for large values of $a$, i.e., high decay rates. A simple

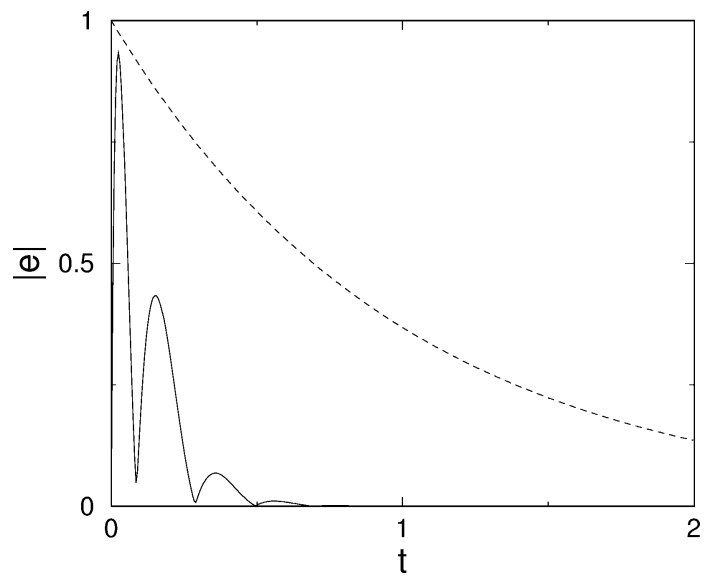

Fig. 2. Numerically computed time evolution of the synchronization error $|\mathbf{e}|$ (full line) for jerky dynamics (2) with $A=0.61$ using the modified BK-coupling scheme (31) and $a=27$. The synchronization error decays faster than $\exp (-t)$ (dashed line).

example for this modified BK-coupling scheme is to substitute

$s=p\left(\mathbf{x}_{d}\right)=a x_{d}+y_{d}+a^{-1} z_{d}+\left|x_{d}\right|$,

$\tilde{s}=p\left(\mathbf{x}_{r}\right)=a x_{r}+y_{r}+a^{-1} z_{r}+\left|x_{r}\right|$

in Eq. (10), respectively. This choice of $p$ satisfies inequalities (28). Since only $p^{x}$ is non-zero we get the estimate $a=24 L_{C}+3 c$. For the piecewise linear jerky dynamics (2) a plot of the numerically calculated error dynamics vs. time is shown in Fig. 2. Since this jerky dynamics is piecewise linear the relation $L_{C}=1$ holds for all subsets $C \subset \mathbb{R}^{3}$ (global synchronization). Requiring a decay of the error that is faster than $\exp (-t)$, implies that $a \geqslant 27$. In the plot in Fig. 2, $a=27$ has been used and one consistently recovers the desired decay of this modified BK-coupling.

\section{Active-passive decomposition (APD)}

In a nutshell, active-passive decomposition (APD) used by Kocarev and Parlitz [5-7] works as follows. Consider two functionally identical dynamical systems, the active system $\dot{\mathbf{v}}_{d}=\mathbf{V}\left(\mathbf{x}_{d}, \mathbf{s}\right)$ and the passive system $\dot{\mathbf{x}}_{r}=\mathbf{V}\left(\mathbf{x}_{r}, \mathbf{s}\right)$. Both systems only differ by the entering drive and response variables $\mathbf{x}_{d}$ and $\mathbf{x}_{r}$, respectively. Otherwise, the functional form of the vector field $\mathbf{V}$ and the additional coupling, the drive 
vector $\mathbf{s}=\mathbf{h}\left(\mathbf{x}_{d}\right)$, that functionally depends only on the drive variables $\mathbf{x}_{d}$, are the same. The error dynamics $\dot{\mathbf{e}}=\mathbf{V}\left(\mathbf{x}_{d}, \mathbf{s}\right)-\mathbf{V}\left(\mathbf{x}_{d}-\mathbf{e}, \mathbf{s}\right)$ linearized about $\mathbf{e}=\mathbf{x}_{d}-\mathbf{x}_{r}=0$ obeys the generally nonautonomous equation $\dot{\mathbf{e}}=D \mathbf{V}\left(\mathbf{x}_{d}, \mathbf{s}\right) \cdot \mathbf{e}$ with $D \mathbf{V}\left(\mathbf{x}_{d}, \mathbf{s}\right)$ being the Jacobian of the vector field $\mathbf{V}\left(\mathbf{x}_{d}, \mathbf{s}\right)$. Obviously, if the specific form of the Jacobian allows for a decay of the error e to zero in the long time limit, synchronization of the active and the passive systems takes place. Since the active system should be the unconstraintly evolving, chaotic system, its vector field $\mathbf{V}\left(\mathbf{x}_{d}, \mathbf{s}\left(\mathbf{x}_{d}\right)\right)$ is just an appropriate decomposition of the original vector field $\mathbf{V}\left(\mathbf{x}_{d}\right)$.

Next, we want to adopt this scheme specifically to chaotic jerky dynamics and, in addition to that, also require that only a purely scalar signal $s(t)$ is transmitted to the passive system. This can be achieved by the following scheme with

$\dot{x}_{d}=y_{d}$,

$\dot{y}_{d}=z_{d}$,

$\dot{z}_{d}=-a x_{d}-b y_{d}-c z_{d}+s(t)$

for the active system,

$\dot{x}_{r}=y_{r}$,

$\dot{y}_{r}=z_{r}$,

$\dot{z}_{r}=-a x_{r}-b y_{r}-c z_{r}+s(t)$

for the passive system, and

$$
\begin{aligned}
s(t) & =s\left(\mathbf{x}_{d}\right) \\
& =J\left(x_{d}, y_{d}, z_{d}\right)+a x_{d}+b y_{d}+c z_{d}
\end{aligned}
$$

for the transmitted signal. Here $a, b$ and $c$ are so far arbitrary real constants that have to be suitably adjusted in order to obtain synchronization. Note that (32) is in fact the dynamical system associated with the jerky dynamics $\dddot{x}=J(x, \dot{x}, \ddot{x})$; the only difference is that the same term, $a x_{d}+b y_{d}+c z_{d}$, has been artificially added and subtracted. Moreover, this coupling scheme differs from BK-coupling by the fact that not only a linear combination of the drive variables $\mathbf{x}_{d}$ enters in the transmitted signal, but also the full dynamical information on the chaotic system, namely the jerk function $J\left(x_{d}, y_{d}, z_{d}\right)=\dddot{x}_{d}$.

As in the previous section, we first apply the scheme to the jerky dynamics (2) with $A=0.61$. In Fig. 3 we

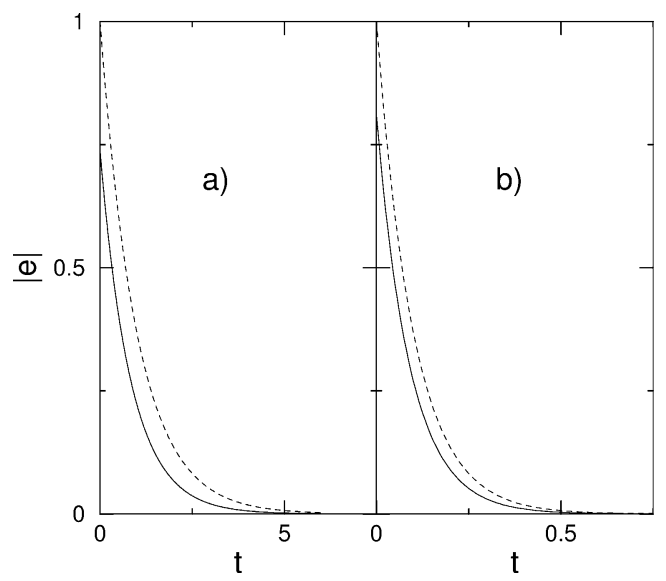

Fig. 3. Numerically computed time evolution of the synchronization error $|\mathbf{e}|$ (full line) for the jerky dynamics (2) with $A=0.61$ using the APD-coupling scheme (32)-(34). The parameters are $a=2.184$, $b=5.06, c=3.9$ in (a) and $a=1320, b=362, c=33$ in (b). The synchronization error decays faster than $\exp (-t)$ in (a) and $\exp (-10 t)$ in (b) (dashed line).

show the time evolution of the modulus of the error dynamics $|\mathbf{e}|=\left|\mathbf{x}_{d}-\mathbf{x}_{r}\right|$ for this model and two representative sets of adjustable parameters $(a, b, c)$, namely $(2.184,5.06,3.9)$ in Fig. 3(a) and $(1320,362,33)$ in Fig. 3(b). In both cases, synchronization of the active and passive system occurs; for the first parameter set the decay is faster than $\exp (-t)$, for the second faster than $\exp (-10 t)$.

The reason why the coupling scheme (32)-(34) effectively leads to synchronization for any (bounded) jerky dynamics can be substantiated by looking at the corresponding error dynamics

$\dot{\mathbf{e}}=A \mathbf{e}$

with the matrix

$\mathrm{A}=\left(\begin{array}{ccc}0 & 1 & 0 \\ 0 & 0 & 1 \\ -a & -b & -c\end{array}\right)$.

Since Eq. (35) constitutes an autonomous linear problem, the error $\mathbf{e}=\mathbf{x}_{d}-\mathbf{x}_{r}$ approaches the stable equilibrium $\mathbf{e}=0$ if $A$ possesses only negative eigenvalues. All the information of the jerk function $J(x, \dot{x}, \ddot{x})$ is hidden in the signal $s=J\left(x_{d}, y_{d}, z_{d}\right)+a x_{d}+b y_{d}+$ $c z_{d}$. Looking at the characteristic equation associated with $A$,

$\lambda^{3}+c \lambda^{2}+b \lambda+a=0$, 
and taking into account that $a, b, c$ are freely adjustable parameters, it is always possible to choose these parameters in a way that all eigenvalues of $A$ are negative and smaller than a given value $-k$ with $k>0$. Denoting the eigenvalues of $A$ by $-\lambda_{1},-\lambda_{2}$ and $-\lambda_{3}$, then the specific selection

$a=\lambda_{1} \lambda_{2} \lambda_{3}$,

$b=\lambda_{1} \lambda_{2}+\lambda_{1} \lambda_{3}+\lambda_{2} \lambda_{3}$

$c=\lambda_{1}+\lambda_{2}+\lambda_{3}$

obviously fulfills Eq. (37). The afore-mentioned representative examples have been selected by this method; in Fig. 3(a) the eigenvalues have been chosen to be $-\lambda_{1}=-1.2,-\lambda_{2}=-1.3$ and $-\lambda_{3}=-1.4$, whereas in Fig. 3(b) $-\lambda_{1}=-10,-\lambda_{2}=-11$ and $-\lambda_{3}=-12$ have been used. This corresponds to the choice $a=$ 2.184, $b=5.06, c=3.9$ and $a=1320, b=362$, $c=33$.

The so far discussed decomposition scheme can also be generalized by using a nonlinear function $G\left(\mathbf{x}_{d}\right)$ in order to complicate the determination of all relevant parameters using autosynchronization [18] and to make the corresponding communication schemes more secure. Then the transmitted signal is given by $s=J\left(\mathbf{x}_{d}\right)-G\left(\mathbf{x}_{d}\right)$ and the differential equations for the drive and response system are determined by

$\dot{x}=y$,

$\dot{y}=z$,

$\dot{z}=G(x, y, z)+s$.

For the corresponding error dynamics of $\mathbf{e}=\mathbf{x}_{d}-\mathbf{x}_{r}$ one obtains

$\dot{\mathbf{e}}=\left(\begin{array}{ccc}0 & 1 & 0 \\ 0 & 0 & 1 \\ G^{x} & G^{y} & G^{z}\end{array}\right) \mathbf{e}$

with the functions $G^{x}, G^{y}$ and $G^{z}$ defined analogously to the $J^{x}, J^{y}, J^{z}$ in Eq. (14) by exchanging the role of $J$ and $G$. Rewriting Eq. (40) as

$\dot{\mathbf{e}}=\mathrm{Ae}+\mathrm{Be}$

with

$\mathrm{A}=\left(\begin{array}{ccc}0 & 1 & 0 \\ 0 & 0 & 1 \\ -a & -b & -c\end{array}\right)$,
$\mathrm{B}=\left(\begin{array}{ccc}0 & 0 & 0 \\ 0 & 0 & 0 \\ G^{x}+a & G^{y}+b & G^{z}+c\end{array}\right)$

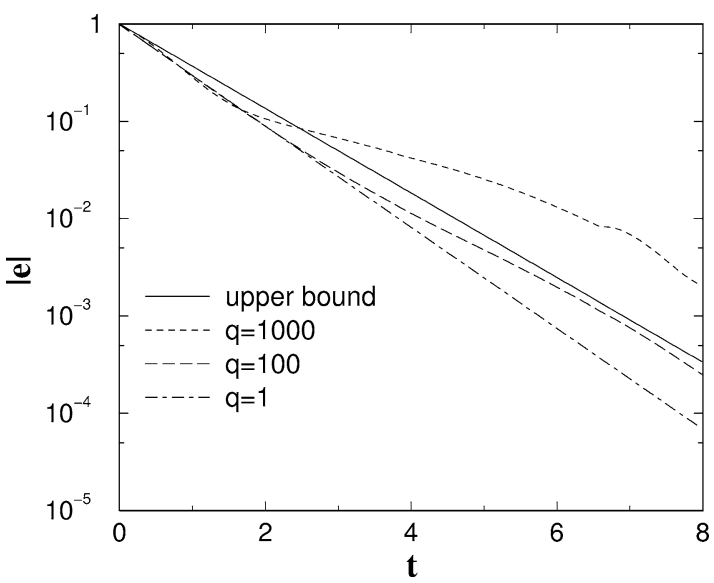

Fig. 4. Numerically computed time evolution of the synchronization error $|\mathbf{e}|$ for the jerky dynamics (2) with $A=0.61$ using the modified APD scheme (39) and (43) for different values of the parameter $q$ in Eq. (43) with $a=2.184, b=5.06, c=3.9$. The synchronization error decays faster than $\exp (-t)$ (solid line) for $q=1$. Even if the parameter $q$ is larger than $1, q=100$, the system synchronize with an error which decays faster than $\exp (-t)$.

we can again adapt the Poincare-Lyapunov technique used in the previous section to obtain estimates of the parameters $a, b, c$ that guarantee synchronization. As a representative example for the outcome of such a modified APD for jerky dynamics, we take as nonlinear function

$G\left(\mathbf{x}_{d}\right)=-a x_{d}-b y_{d}-c z_{d}+q\left|x_{d}\right| / 1000$

with $a=2.184, b=5.06, c=3.9$, the free parameter $q$ and the jerk model (2). Using the PoincareLyapunov technique, one can show that a decay of the modulus of the error $|\mathbf{e}|$ which is faster than $\exp (-t)$ is guaranteed for $q \leqslant 1$. As corresponding numerical calculations of $|\mathbf{e}|$ based on Eqs. (39) and (43) for different values of $q$ (shown in Fig. 4) exemplify, the bound for exponential decay proportional to $\exp (-t)$ is rather crude and even for $q=100$ still fulfilled. Only for larger values of $q$, e.g., $q=1000$, there is a significant deviation from an exponential decay.

\section{Conclusion}

Using a variety of different well-known schemes, we have investigated if and under what circumstances elementary flows in form of jerky dynamics can be 
synchronized. Most remarkably, we have shown that the BK-coupling scheme and the APD decomposition can be used for that purpose for any functional form of a chaotic jerky dynamics. By using a specific, rather simple form of the $\mathbf{B K}^{\mathrm{T}}$ matrix or a specific selection of the eigenvalues of the error matrix in the APD case, we were able to derive estimates for the corresponding decay of the synchronization error. We also exemplified our findings by using the piece-wise linear chaotic model (2). Due to its simple functional form, its realization as an electronic circuit does not involve nonlinear elements, but only an inverter, leading to a highly accurate analog representation of the dynamical behavior for the whole chaotic control parameter range and, therefore, serving as an ideal candidate for potential applications of synchronization to private communication.

So far our discussion has focused on the synchronization of chaotic jerky dynamics. In a far wider context, however, our investigation is directly applicable to any three-dimensional dynamical system that can be transformed to a jerky dynamics. Examples for such systems are the jerky dynamics of the $y$-component of the Rössler model [10] and the variety of jerky dynamics [11] corresponding to Sprott's algebraically simple chaotic models [19].

\section{Acknowledgements}

The authors acknowledge support by the "Graduiertenkolleg: Nichtlineare Probleme in Analysis, Geome- trie und Physik" (GRK 283) financed by DFG and the state of Bavaria.

\section{References}

[1] L.M. Pecora, T.L. Carroll, Phys. Rev. Lett. 64 (1990) 821.

[2] L.M. Pecora, T.L. Carroll, G.A. Johnson, D.J. Mar, J.F. Heagy, Chaos 7 (1997) 520.

[3] K.M. Cuomo, A.V. Oppenheim, Phys. Rev. Lett. 71 (1993) 65; K.M. Cuomo, A.V. Oppenheim, S.H. Strogatz, Int. J. Bif. Chaos 3 (1993) 1629.

[4] J.H. Peng, E.J. Ding, M. Ding, W. Yang, Phys. Rev. Lett. 76 (1996) 904

[5] Ch.W. Wu, L.O. Chua, Int. J. Bif. Chaos 3 (1993) 1619.

[6] L. Kocarev, U. Parlitz, Phys. Rev. Lett. 74 (1995) 5028.

[7] U. Parlitz, Phys. Rev. Lett. 76 (1996) 1232.

[8] H.P.W. Gottlieb, Am. J. Phys. 64 (1996) 525.

[9] J.C. Sprott, Phys. Lett. A 228 (1997) 271; J.C. Sprott, Am. J. Phys. 65 (1997) 537.

[10] S.J. Linz, Am. J. Phys. 65 (1997) 523; S.J. Linz, Am. J. Phys. 66 (1998) 1109.

[11] R. Eichhorn, S.J. Linz, P. Hänggi, Phys. Rev. E 58 (1998) 7151 ;

R. Eichhorn, S.J. Linz, P. Hänggi, Chaos Solitons Fractals 13 (2001) 1.

[12] S.J. Linz, J.C. Sprott, Phys. Lett. A 259 (1999) 240.

[13] J.C. Sprott, Phys. Lett. A 266 (2000) 19; J.C. Sprott, Am. J. Phys. 68 (2000) 758.

[14] S.J. Linz, Phys. Lett. A 275 (2000) 204.

[15] J.M. Malasoma, Phys. Lett. A 264 (2000) 383.

[16] Ö. Morgül, E. Solak, Phys. Rev. E 54 (1996) 4803.

[17] F. Verhulst, Nonlinear Differential Equations and Dynamical Systems, Springer, Berlin, 1990, Chapter 6.1.

[18] U. Parlitz, Phys. Rev. Lett. 76 (1996) 1232.

[19] J.C. Sprott, Phys. Rev. E 50 (1994) R647. 